\title{
TCAR: The Trans-Atlantic Cooperation for a state-of-the-art AGS Radar
}

\author{
Peter Hoogeboom ${ }^{\# * 1}$, Stéphane Buvry ${ }^{\$ 2}$, Andreas Löhner ${ }^{\& 3}$ \\ ${ }^{\text {\#}}$ TNO Defence, Security and Safety, The Hague, Netherlands \\ peter.hoogeboom@tno.nl \\ *Delft University of Technology, IRCTR, Delft, Netherlands \\ ${ }^{\$}$ TCAR Industries GmbH, Brussels, Belgium \\ ${ }^{2}$ stephane.buvry@fr.thalesgroup.com \\ ${ }^{\circledR}$ TCAR Industries GmbH, Ulm, Germany \\ 3andreas.1oehner@eads.com
}

\begin{abstract}
In view of the NATO Air-to-Ground Surveillance requirement and the results of the 2004 Feasibility Study on the development of an advanced SAR/MTI radar system, the TCAR industries, represented by a group of industries from 6 nations (FR, GE, IT, NL, SP, USA), have been tasked by the NATO AGS Prime Contractor to conduct a 6-month Risk Reduction Study. This study and follow-on activities have led to the current readiness level for a Design \& Development contract.

The architectural design of the system is based on knowledge from currently ongoing and legacy radar programs in these countries and the NATO AGS requirements. To perform the program the radar industries have founded the TCAR-Industries GmbH, which is a joint venture of these companies.

A programmatic and technical overview of the TCAR program will be presented in this paper. Furthermore, a discussion is presented on global requirements, platform configuration, technical approach to simultaneous SAR and MTI operation, the global radar configuration, processing aspects and radar mode developments.
\end{abstract}

\section{INTRODUCTION}

The North Atlantic Treaty Organization (NATO) or members thereof, are considering the development of an airborne ground surveillance radar system, known as the NATO Alliance Ground Surveillance (AGS) System. The operational need for an AGS capability, as defined by the NATO Staff Requirement (NSR), is based on a military requirement stated by the NATO Military Authorities (NMA) and a subsequent tasking by the North Atlantic Council (NAC) to the conference of National Armament Directors (CNAD) in 1993 and reconfirmed by the Reinforced NAC in September 2001.

The AGS System will be a NATO owned and operated capability, consisting of radar-equipped aircraft, Operation and Control stations, datalinks and ground stations. The advanced radar system TCAR (Transatlantic Cooperative AGS Radar) [1] will form the heart of the system. The radar development will be led by the TCAR-Industries $\mathrm{GmbH}$, which subcontracts the national lead industries of the 6 TCAR nations. The TCAR program will leverage technology from existing programs, primarily the European SOSTAR (Stand-
Off Surveillance Target Acquisition Radar) [2, 3] and similar US radar programs.

In the first quarter of 2005, NATO initiated procurement strategy for a 6-month NATO AGS System Risk Reduction Study (RRS) which culminated in an award to the NATO AGS Prime Contractor on 28 April 2005. A subsequent award was made by the Prime to the TCAR Team in May with EADS providing management.

The TCAR industry team successfully completed the RRS on time.

The Design and Development (D\&D) Phase, the Engineering Manufacturing Development (EMD) Phase and Production Phases will be executed by the TCAR-Industries $\mathrm{GmbH}$. This joint venture company (JVC) was established in 2006 in Germany. To optimize the support to the customer the JVC has established in the beginning of 2007 an office in Brussels.

The TCAR radar sensor will satisfy AGS Core requirements as specified in the AGS NSR and the Concept of Operations (CONOPS), and satisfy as well, the need for national applications of the TCAR technologies. The radar requirements are derived from the system requirements, which are detailed in the Technical Requirement Document (TRD) from the NATO. The TCAR system will be an advanced airborne ground surveillance sensor based on modern AESA (Active Electronically Scanned Array) antenna technology.

The objective of the TCAR program is the development and manufacturing of this next generation SAR Imaging/ MTI radar sensor, and its integration into a manned airborne platform, including its support in operation, to satisfy the AGS requirements. The TCAR concept is based on a modular, scalable and open system architecture. The modularity and scalability ensure the adaptation to the AGS manned platform, the Airbus A321 Midsize-Jet, as well as to other programs, e.g. for national use.

The TCAR is an advanced, side-looking, wide area surveillance (WAS), long range, multi-mode radar. The radar is characterized by:

- An X-band, active electronically scanning array (AESA), 
- Low antenna side-lobes,

- High reliability and graceful antenna degradation,

- High effective radiated power (ERP) from production transmit/receive modules (TRMs),

- Instantaneous, wide bandwidth,

- Concurrent SAR and MTI-functions,

- Latest generation of COTS digital signal processors,

- Built-in calibration and measurement.

\section{TCAR CONSORTIUM OVERVIEW}

The US and European governments (Germany, France, Italy, Spain and The Netherlands) tasked their industries with AESA experience to initiate an AGS Radar (TCAR) Feasibility Study. The TCAR study was declared "feasible" by the governments in July 2004.

Subsequently, NATO initiated the 6-month RRS in May 2005, while, with EADS in the management role, the management and commercial teams were completing the details and formalizing the JVC.

The partners in the TCAR Industries $\mathrm{GmbH}$ are the following US and European radar industries:

- Dutch Space

NL

- European Aeronautic Defence and GE Space (EADS)

- Galileo Avionica

IT

- Indra Sistemas

SP

- Northrop Grumman

US

- THALES Airborne Systems

FR

TCAR work share for the D\&D and EMD Phases has been divided to support national and industrial goals for the program. International cooperation is evident with all team members supporting System Engineering and Sensor System Integration and Test (I\&T). In addition, US and European companies will participate in development of the software operating system (known as the Radar Operating Services ROS) which will facilitate overall software development. The development of the radar modes will be under the responsibility of individual companies. The Antenna will be designed, fabricated and built by the 5 European industries while the US team will provide the COTS-based Processor and the Central Electronics Suite.

\section{RADAR ARCHITECTURE}

The architecture proposed for the TCAR is based on a modular, scalable and open radar system design. In characterizing the system, there are several requirements that drive the architecture. Some key points are:

- A multi-beam capability and a high aperture power product to enable rapid revisit rate of moving targets in a large area at long range,

- Independent hardware and a high performance processor to enable concurrent modes, e.g. SAR and MTI with a large coverage rate

- A large antenna configured with multiple receivers enables high positioning accuracy and supports a small Minimum Detectable Velocity (MDV) of moving targets,
- Time delay networks and small element spacing in the antenna to allow for large squint angles, in order to cover large areas,

- Wide-band waveform generators, receivers and other components to allow for high resolution in all modes.

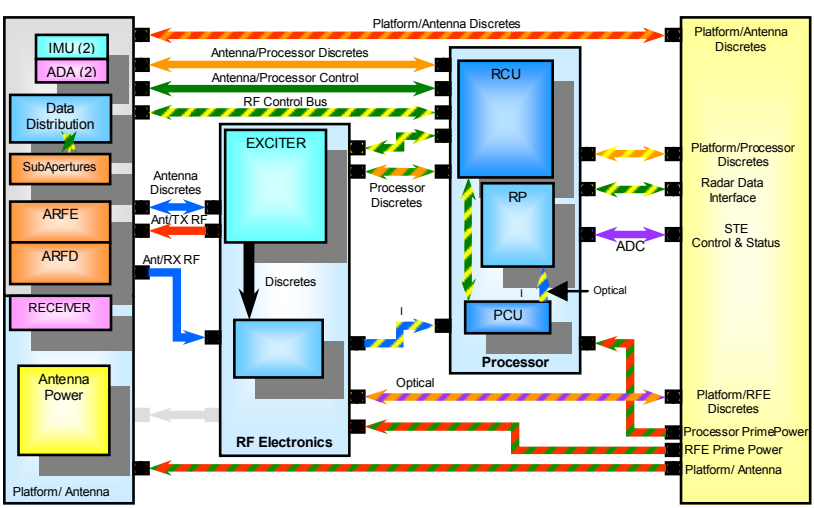

Figure 1: Overview of the radar Prime Architectural Elements (PAEs)

The application of scalable antenna design techniques enables the re-use of the technology base in TCAR radar designs for other, e.g. national programs, allowing also the re-use of the same T/R modules.

The scalable radar system hardware architecture, shown in Figure 1, consists of:

- Antenna Group (left),

- Central Electronics Group (mid),

- Radar Processing Group (right).

These elements are internally connected via a radar control Local Area Network (LAN). Data is internally transported via specific interfaces to accommodate fast transport requirements (not shown in the figure). Connections to the AGS Mission Subsystem will be established via high speed interfaces for radar output data and for radar control, tasking and navigation data.

The Antenna Group, the Central Electronics Group and the Radar Processing Group are described in more detail in the following sections.

\section{A. Antenna Group}

The demanding requirements on radar performance necessitate the use of a long antenna. For the manned aircraft, it is in excess of 6 meters.

The antenna will be developed and built by the European companies as mentioned in Section II. This includes the realization of the Transmit/Receive Modules.

The radar antenna will be an AESA, mounted with a one axis roll gimbal. This gimbal orientation allows radar pointing to the left or the right side of the platform. In azimuth, an electronically scanned beam achieves a wide angular coverage. In elevation, the electronic scan capability allows full illumination of the Ground Reference Coverage Area (GRCA).

A high transmit power is achieved by the use of a large number of TRMs. The AESA inherently supports high system availability enabled by graceful degradation of radar 
performance. Liquid cooling is applied in the Antenna Group to support the high power dissipation requirements.

To precisely determine the position and orientation of the antenna for each transmitted pulse and receive window, a Precise Orbit Estimation System (POES) will be developed. Information from an Inertial Measurement Unit (IMU), from GPS and from accelerometers on the antenna group is combined in a real-time Kalman filtering process. The resulting state vectors are outputted to the Radar Control Unit.

The Antenna Group is composed of:

- On-Axis and Off-Axis Antenna structure and frame,

- Antenna-panels, planks (basic building block of the scalable AESA antenna) with a predefined elevation structure and sub-arrays,

- Separate manifolds for transmit and certain receive modes,

- Beamformer, RF interface and RF converter,

- Harness, Beam Steering Computer, Time Delay Networks, DC power supply and IMU,

- Antenna Gimbal and servo.

\section{B. Central Electronics Group}

The Central Electronics (CE) Group (Receiver/ Exciter) is based on a modular and configurable design, in order to provide opportunities for technology insertion for future applications and upgrades. The CE supports a variety of modes including concurrent SAR/MTI modes.

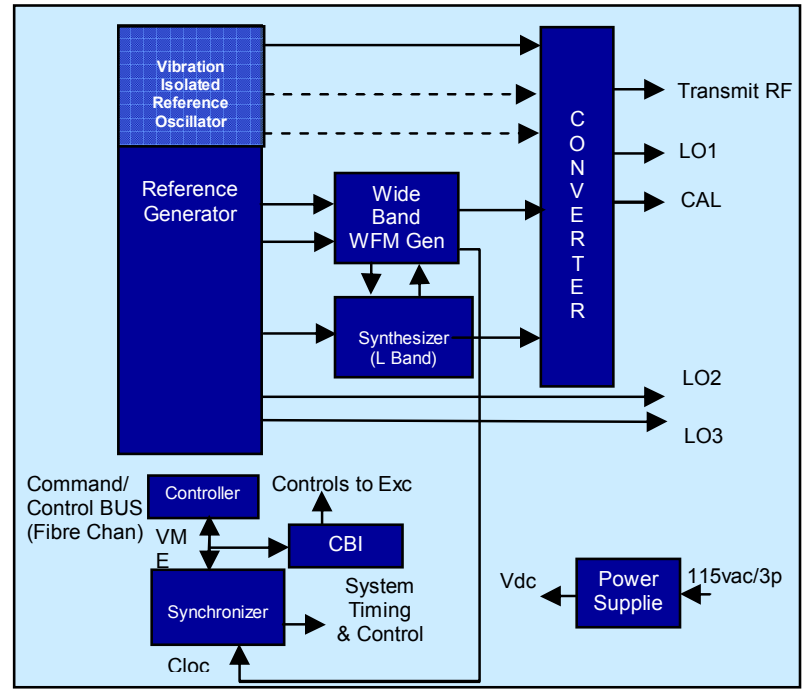

Figure 2: Exciter Block Diagram

The Central Electronics Group includes:

- The Exciter generates the waveforms required for the
SAR, MTI, HRR (High Range Resolution) and simultaneous modes. Furthermore, all reference Local Oscillator signals for up, down conversion and timing base will be generated and distributed as shown in the Exciter Block Diagram in Figure 2.

- The multi-channel digital receiver supports the down conversion with fixed LO and de-ramping techniques. Multi-beam-on-receive techniques are also supported. The receivers support advanced signal pre-processing, including digital I/Q conversion, digital downconversion and data formatting as shown in the Receiver Block Diagram in Figure 3. Also highlighted is the proposed On-Axis/Off-Axis configuration.

- Calibration support to adjust gain and noise levels in the AGC attenuators.

- The power conditioner for filtering and providing the power for the Antenna Group.

- Collection of information from the POES in the Antenna Group and distribution of these data to the Radar Processing Group.

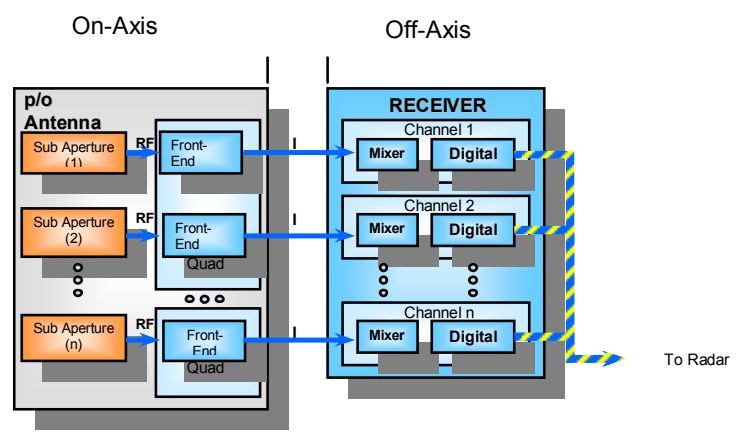

Figure 3: Receiver Block Diagram

\section{Radar Processing Group}

The Radar Processing Group includes:

- The Radar Management and Control Processor,

- The COTS based Radar Signal Processor

The Processor performs: Radar Control, Pulse Compression and Radar Data/Signal Processing functions. It accepts I/Q data from the Receivers, applies mode independent adaptive signal processing algorithms and outputs the required radar products to the platform mission computer via the Radar Data Interface.

Figure 1 shows the control data flow within the radar and between the radar and the AGS Mission Sub-system.

The Radar Management and Control Processor will manage proper operation of the radar subsystem and handle the control and tasking between the Radar and the AGS Mission Subsystem. 


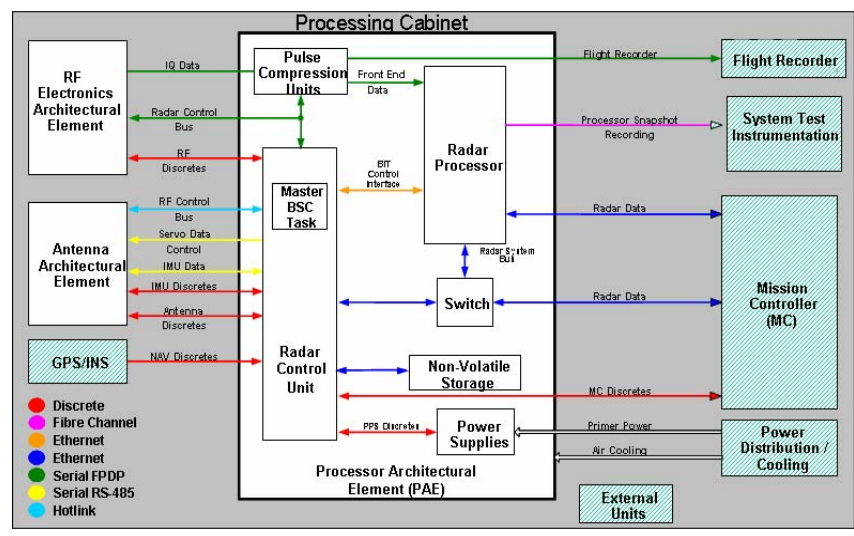

Figure 4: Radar Processor Architectural Element (PAE) Block Diagram with Interfaces

The Block Diagram for the Radar Management and Control Unit is shown in Figure 4. The Processor will perform the following operations within the radar:

- Verify that the actions commanded by the AGS Mission Sub-system, to be performed by the radar, are properly received and understood.

- Distribute the orders received from the AGS Mission Sub-system to the different radar components involved in their execution.

- Generate the proper commands for the rest of the radar elements, including beam control and antenna pointing and selection of the proper waveform parameters for the radar modes to be applied at the Central Electronics Group.

- Collect status, built-in test reports and relevant radar diagnostic information from the different radar elements. This information is transferred to the AGS Mission Sub-system.

- Supervise data flow between the AGS Mission Subsystem and the Radar.

- Monitor the requested radar tasks to insure that they are being performed according to the expected operational capabilities of the radar.

- Perform periodical housekeeping and monitoring tasks of the different radar activities.

- Collect GPS and navigation data from all relevant platform sensors and provide them to the radar system.

A separate radar control capability will enable radar standalone operation (e.g. during radar integration, test, radar validation and in-field maintenance). This radar control capability can generate radar tasks.

The radar signal processor handles digitized data that will come from the receiver together with all the radar setup information (time, PRF, frequency, etc.). These data are processed in real time. Separate processing sections take care of the concurrently available SAR and MTI data streams.

The COTS based Radar Signal Processor will process all the digital channels implemented at the receivers. This processor will provide the different radar products required by the AGS Mission Sub-system.
Due to the high data rate involved in the signal processing, it is expected that the data will be sent over a dedicated highspeed data network to the AGS Mission Sub-system.

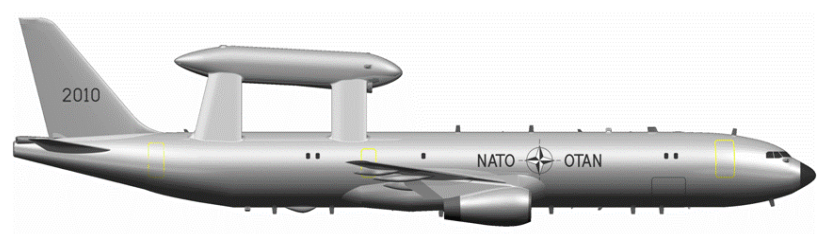

Figure 5: Airbus A321 Platform

Parameters:

- Maximum Altitude: 11,900 meters (39,000 feet)

- Optimum Orbital Speed: 477 to 503 knots (Mach 0.74 to 0.78)

- Range: 10.3 hours (un-fueled), 30.6 hours (refueled)

- Maximum Payload: 22,734 kilograms (50,119 pounds)

\section{PLATFORM}

The Airbus A321 Mid-Size Jet is the selected NATO AGS manned platform. Figure 5 shows the "top mount" configuration for the TCAR. The TCAR Team will support the Prime Contractor in conducting system, platform and sensor trade studies to achieve the best value configuration.

Most parts of the Central Electronics Group and the Radar Processing Group are situated in racks in the cabin, whereas most parts of the Antenna Group (i.e. the radiating unit) will be situated in the top-mounted pod. The Radar is designed to handle the considerable distances between the units with minimal performance losses.

\section{RADAR MODES}

The primary mission of the AGS platform is to provide the complete evolving picture of the battlefield or surveillance area over time. To accomplish this, the AGS System identifies and tracks all moving ground targets while providing high resolution imagery to the platform operators. This information can then be disseminated to the various responsible parties throughout the NATO command structure. The TCAR radar supports the tracking and identification of moving vehicles by a variety of radar modes. The major advancement of the TCAR radar over similar legacy systems is the ability of the radar to simultaneously collect GMTI detections and SAR imagery. This will allow NATO Commanders to see and monitor not only the moving targets within the Area of Interest, but to see the underlying infrastructure simultaneously.

The various radar modes will be developed by individual companies within the TCAR Consortium. The enabling technology behind the ability to co-operatively develop Radar Modes by individual companies is the robust design of the Radar Operating Services (ROS).

The ROS is a layered, modular, scalable architecture that is designed to support the operation of radar modes in the TCAR Signal Processor and simplify future growth. In order to maximize flexibility, it is designed to operate on common Signal Processor architectures by isolating the operating system and hardware specific differences; it can be 
commanded by external or internally generated Radar Service Requests (RSR) and can be configured by mission parameters.

The ROS provides:

- The entire infrastructure required by the radar modes to perform their required signal processing in the TCAR Radar Processor

- Arbitration of front-end use to balance operation and minimize waste

- Support of high- and low-latency collection and processing priorities for different mode sequences and concurrent operation of radar modes

- The memory management to prevent resource contention

- The fault tolerance through the use of dynamic node allocation allows operation to continue even if some nodes fail.

Dynamic node allocation is achieved by the use of Virtual Clusters. A Virtual Cluster is a group of individual dynamic processors working together to perform mode signal processing. The processors are not necessarily within the same vicinity. Physical allocation of each Virtual Cluster will be dynamically assigned. Assignment is based on the number of processors requested by the mode and the processors available when the mode is scheduled. The Virtual Cluster methodology supports any signal processing method(s) that the radar modes may choose to utilize; pipeline processing, parallel processing, round-robin processing, any combination thereof, etc.

ROS libraries provide uniform methods for inter-processor communication and signal processing tasks within the proposed TCAR radar processor. Mode software is protected from any vendor-specific Application Programming Interfaces (APIs), resulting in increased software portability and maintainability. Data Communications and Signal Processing performance are the two biggest areas of concern for mode performance. ROS employs an open architecture approach to standardizing both data communications and signal processing.

The following operational radar modes will be implemented:

- Moving Target Indicator (MTI): The Ground Moving Target Indicator (GMTI) mode is capable of detecting moving ground targets, and ground-based stationary rotating antennas. The GMTI mode is also capable of detecting moving surface targets in lakes, rivers, inshore waterways and in the littoral region. Individual targets within convoys or groups will be resolved with sufficient accuracy for targeting. A high resolution ground MTI mode (High Range Resolution Wide Area Surveillance (HRR WAS)), a medium resolution ground MTI mode and a medium resolution maritime MTI mode will be provided. A wide area is covered with these MTI modes with a high revisit rate. The plots generated by the MTI modes will be delivered to the AGS Mission Sub-system, which will perform the target tracking. Sample MTI data, projected on a map is displayed in Figure 6.

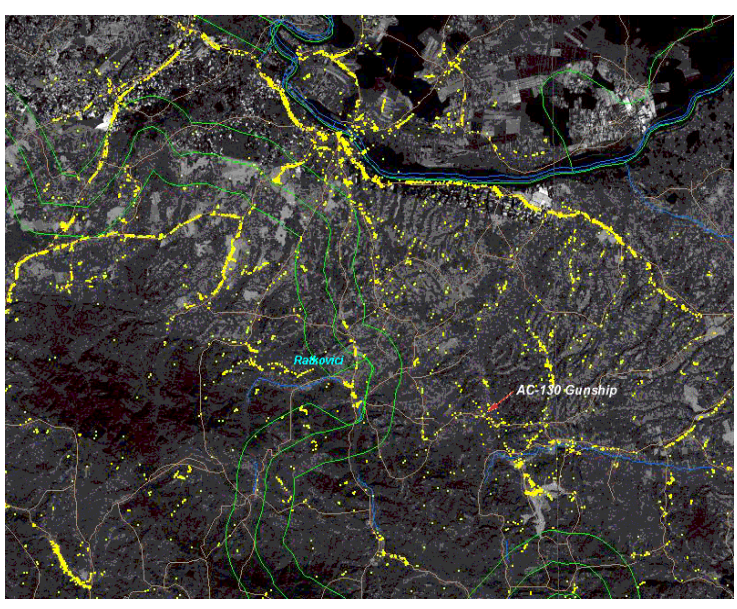

Figure 6: Sample MTI Data

- Classification modes: The classification modes (terrestrial ISAR, maritime ISAR, HRR and Spectral Classification (SPC)) are used to generate data to support the classification of designated targets. High resolution images of vehicles, running in a curve, will be generated by the terrestrial ISAR mode. Vessels will be imaged by the maritime ISAR mode. In HRR mode high range resolution profiles of designated targets are generated. The SPC mode provides Doppler spectra of designated targets. The classification data will be provided to the AGS Mission Sub-system to support the ability to discriminate between wheeled and tracked vehicles, and identify slow flying helicopters and ground rotating antennas.

- Open Sea: The Open Sea mode is used for vessel detection in the open sea and will operate in high sea states.

To provide high resolution imagery of the Area of Interest, the following Synthetic Aperture Radar Modes will be implemented:

- Swath SAR: The Swath SAR mode is used to image long-range swaths at selected image resolutions for the surveillance of fixed targets.

- Spotlight SAR: The Spotlight SAR mode is used for imaging small areas at selected high resolutions to provide detailed images of the targets. Sample Spotlight SAR imagery is shown in Figure 7, which is gathered with the European SOSTAR-X in Spot SAR mode [4]. 


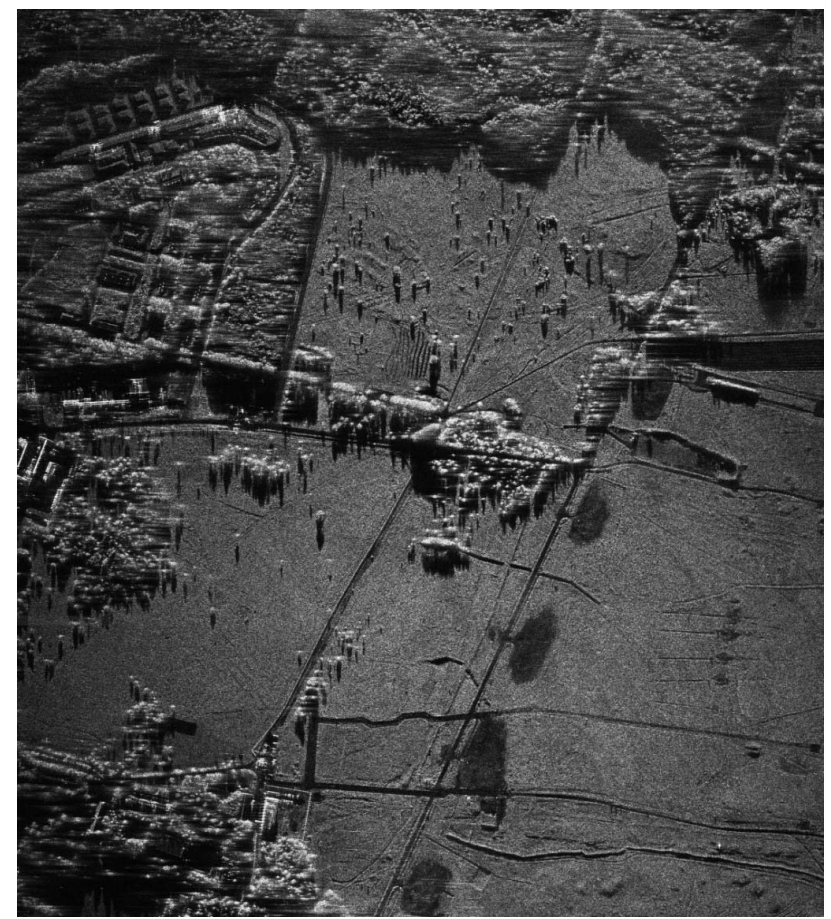

Figure 7: SOSTAR Spotlight SAR Image recorded during test flight in The Netherlands, July 2006 (Courtesy SOSTAR GmbH)

\section{DEVELOPMENT APPROACH}

The Feasibility Study continues to be the foundation for the TCAR Program. This basis was further enhanced by the RRS, which addressed programmatic details in Risk Management, Integrated Program Costs and Integration of TCAR with the NATO AGS System Program Plans, Technology Release and Export Licensing.

The TCAR program is in the final preparation phase for the D\&D Contract Award which is expected in CY2007. The program will start with the Design and Development Phase. A detailed design will be made by a joint system engineering team. TCAR will be based on knowledge and technology from US and European AESA programs. The D\&D Phase will be followed by the Engineering Manufacturing and Development (EMD) Phase, in which the first radar will be built up and the performance will be verified. The radar sensor will, as much as possible, be developed, integrated, qualified and flighttested concurrently with the Prime Contractor.

The first radar will be flight-tested on the target aircraft, the A321 Mid-size jet.

The development of software for the radar modes occurs in parallel with the hardware development. A spiral approach with three software builds is proposed, where the single modes are delivered first, followed by the concurrent modes. Based on test flight results and further development, new versions will become available. The concurrent modes will be tested after the successful completion of the single mode validation.

The Production Phase will start under a subsequent contract.

\section{CONCLUSION}

In this paper the design of this state-of-the-art SAR/MTI radar for ground surveillance has been presented. Under the lead of the TCAR Industries $\mathrm{GmbH}$ this radar will be developed and produced by 6 industries and 1 institute of 6 nations and based on technology and knowledge from US and European AESA programs. The radar is designed at the request of 6 NATO countries and should provide NATO and the nations with an initial state-of-the-art Ground Surveillance capability in the year 2012 .

\section{ACKNOWLEDGMENT}

The authors wish to acknowledge the assistance and support of all those who contributed to the TCAR Feasibility Study and the Risk Reduction Study. They specifically acknowledge the continued support of their government representatives throughout the study and in many stimulating discussions.

\section{REFERENCES}

[1] Hoogeboom, P., H. Hommel, G. Canafoglia, J.Hausle, J. Hubeek, L. Lopez, R. Nachman, J. Neuville, "TCAR, the Transatlantic Cooperative AGS Radar: a programmatic and technical overview," in Proceedings 2004 EURAD, 18 - 19 October 2004, Amsterdam, The Netherlands, pp. 145-148, ISBN 1-58053-993-9.

[2] Hoogeboom, P., E. Herpfer, P. Fournet, G. Canafoglia, A. de Carvajal, C. Hofkamp, "SOSTAR, A European system for airborne ground surveillance", in Proceedings International symposium on Information Superiority, 24 - 26 September 2001, Paris, France, 4 pages, published on CD-ROM

[3] Hoogeboom, P., D. van Halsema, E. Herpfer, F. Martin, P. Fournet, D. Perthuis, "SOSTAR-X, a high performance Radar Demonstrator for Airborne Ground Surveillance", in Proceedings EUSAR 2000, 23-25 May 2000, Munich, Germany, pp. 825-827, ISBN 3-8007-2544-4.

[4] Kirscht, M., Lang-Schnee, F., "On-Board Multi-Computer SAR Processor for Long Range Reconnaissance", in Proceedings EUSAR 2002, June 2002, Köln, Germany, pp. 421-424. 